\title{
IMAGES OF HARMONIC MAPS WITH SYMMETRY
}

\author{
THOMAS K. K. AU \& TOM Y. H. WAN
}

\begin{abstract}
We show that under certain symmetry, the images of complete harmonic embeddings from the complex plane into the hyperbolic plane is completely determined by the geometric information of the vertical measured foliation and is independent of the horizontal measured foliation of the corresponding Hopf differentials.
\end{abstract}

In this paper, we find a new explicit relation between the image of harmonic embeddings, with certain symmetry, from the complex plane $\mathbb{C}$ into the hyperbolic plane $\mathbb{H}^{2}$ and the metric of the associated $\mathbb{R}$-tree of the corresponding vertical measured foliation of the Hopf differentials. Unlike in the case of compact surfaces, holomorphic quadratic differentials cannot be determined by the vertical measured foliation only. So it is kind of surprising for us to find that the image set of the corresponding complete harmonic embedding is completely determined by the vertical measured foliation and is independent of the geometric information of the horizontal measured foliation.

The symmetry condition that we consider is as follow. We assume that the harmonic embedding $u$ from $\mathbb{C}$ into $\mathbb{H}^{2}$ is invariant under the group $\mathbb{Z}_{k}$ by rotations and its image is an ideal polygon with $2 k$ vertices for any integer $k \geq 2$. This is the next nontrivial case after the case of $\mathbb{Z}_{2 k}$ symmetry which gives harmonic embeddings with regular polygonal images. This condition can be regarded as $u$ having half of the symmetry of a regular polygon.

The symmetry assumption implies that the Hopf differentials are of the form $\left[z^{2 m}-(a+\right.$ $\left.i b) z^{m-1}\right] d z^{2}$ for $a+i b \in \mathbb{C}$. For a generic holomorphic quadratic differential in this family, the associated $\mathbb{R}$-tree has $m+1$ finite edges of equal length given by $\nu=\pi|b| /(2(m+1))$. We will show that

Theorem 1. Let $u: \mathbb{C} \rightarrow \mathbb{H}^{2}$ be the unique (up to equivalence) complete orientation preserving harmonic embedding associated to a quadratic differential equivalent to $\left[z^{2 m}-(a+i b) z^{m-1}\right] d z^{2}$. Then, up to isometry, the image $u(\mathbb{C})$ is the interior of the ideal polygon with vertices given 2000 Mathematics Subject Classification. Primary 53C43.

The second author is partially supported by Earmarked Grants of Hong Kong CUHK4291/00P. 
by $\left\{1, e^{i \alpha}, \omega, \omega e^{i \alpha}, \ldots, \omega^{m}, \omega^{m} e^{i \alpha}\right\}$ in the unit disc model of $\mathbb{H}^{2}$, where $\omega=e^{2 \pi i /(m+1)}$,

$$
\alpha=\alpha_{m}(\nu)=2 \tan ^{-1}\left(\frac{\sin (\pi /(m+1))}{\cos (\pi /(m+1))+e^{2 \nu}}\right),
$$

and $\nu=\pi|b| /(2(m+1))$ is the common length of the finite edges of the $\mathbb{R}$-tree associated to the quadratic differential given by Lemma 1.1.

In this paper, a harmonic embedding $u$ is called complete if its $\partial$-energy metric $\|\partial u\|^{2}|d z|^{2}$ is a complete metric on $\mathbb{C}$, where $z$ is the standard complex coordinate on $\mathbb{C}$.

The result is related to the work of Shi and Tam [7]. The facts that complete harmonic embeddings from $\mathbb{C}$ to $\mathbb{H}^{2}$ are parametrized by Hopf differentials [8, 9] and the images are determined by the asymptotic behaviors of the harmonic embeddings [1, 2, 3], suggest the following problem as a step toward Schoen's conjecture [6] on the nonexistence of harmonic diffeomorphism from the complex plane to the hyperbolic plane: Suppose that $u$ is a complete orientation preserving harmonic embedding with polynomial Hopf differential $P(z) d z^{2}$, is it possible to find explicit relation between the coefficients of $P(z)$ and the vertices of $\overline{u(\mathbb{C})}$ ? For this problem, they showed that, up to isometry, the image of a complete orientation preserving harmonic embedding from the complex plane into the hyperbolic plane is a regular ideal polygon if its Hopf differential is given by $\left(z^{2 m}-a z^{m-1}\right) d z^{2}$ for some real number $a$. This is the first nontrivial example of a family of harmonic maps (for fixed $m$ ) with identical images.

It is obvious that our result is a generalization of that of Shi-Tam. However, the method is quite different. In [7, the authors studied the asymptotic behavior of the image of the harmonic maps along euclidean rays to infinity. Our approach adopts more geometric properties of the Hopf differential, especially those related to the metric information of the $\mathbb{R}$-tree associated to the vertical measured foliation of the Hopf differential. The relationship between the asymptotic behavior of harmonic maps and the associated $\mathbb{R}$-trees has been studied by Minsky [5] and Wolf 10, 11, 12, independently. In these works, the asymptotic behavior of a sequence of harmonic maps on a compact surface with energy (or the norm of the Hopf differential) going to infinity was studied. In our case, instead of a sequence of maps, we are interested in the asymptotic behavior of harmonic maps on a complete noncompact surface as in 3 . In particular, the asymptotic behavior of the length of the image of a horizontal trajectory near 
infinity was studied. More precisely, it was shown that the image of a horizontal trajectory is asymptotic to a geodesic; and the difference between the lengths of this image and the asymptotic geodesic is actually tending to zero as the $\Phi$-distance is going to infinity.

The arrangement of this paper is as follows. In Section 11 we will give a brief description of harmonic maps, its Hopf differentials and the geometric information of the $\mathbb{R}$-trees associated to the Hopf differentials. Then we will study the asymptotic behavior of the image of horizontal trajectories in Section 2. Finally, we prove our main result in Section 3 ,

\section{BACKGROUND}

1.1. Harmonic maps between surfaces. Let $M$ and $N$ be oriented surfaces with metrics $\rho^{2}|d z|^{2}$ and $\sigma^{2}|d u|^{2}$, where $z$ and $u$ are local complex coordinates of $M$ and $N$, respectively. A $C^{2}$ map $u$ from $M$ to $N$ is harmonic if and only if $u$ satisfies

$$
u_{z \bar{z}}+2(\log \sigma(u))_{u} u_{z} u_{\bar{z}}=0
$$

The Hopf differential $\Phi=\phi(z) d z^{2}$ of a map $u$ between these surfaces is defined by $\phi(z)=$ $\sigma^{2}(u(z)) u_{z}(z) \bar{u}_{z}(z)$. If $u$ is harmonic, then it is well-known that $\Phi$ is a holomorphic quadratic differential on $M$.

The $\partial$-energy density and $\bar{\partial}$-energy density of $u$ are defined by

$$
\|\partial u\|^{2}=\frac{\sigma^{2}(u)}{\rho^{2}}\left|u_{z}\right|^{2} \quad \text { and } \quad\|\bar{\partial} u\|^{2}=\frac{\sigma^{2}(u)}{\rho^{2}}\left|u_{\bar{z}}\right|^{2} .
$$

In terms of the $\partial$-energy density and $\bar{\partial}$-energy density, the energy density and Jacobian of $u$ can be written as

$$
e(u)=\|\partial u\|^{2}+\|\bar{\partial} u\|^{2} \quad \text { and } \quad J(u)=\|\partial u\|^{2}-\|\bar{\partial} u\|^{2} .
$$

In this paper, we are interested in the case that $M=\mathbb{C}, N=\mathbb{H}^{2}$, and that $u: \mathbb{C} \longrightarrow \mathbb{H}^{2}$ is an orientation preserving open harmonic embedding. In this case, the Jacobian is strictly positive, i.e., $J(u)>0$, and hence $\|\partial u\|^{2}>0$. Therefore, one can consider the $\partial$-energy metric $\|\partial u\|^{2}|d z|^{2}$ on the complex plane $\mathbb{C}$. As mentioned in the introduction, $u$ is called complete if its $\partial$-energy metric $\|\partial u\|^{2}|d z|^{2}$ is a complete metric on $\mathbb{C}$. As the completeness is only defined 
for orientation preserving $u$, the term complete harmonic open embedding implies implicitly that the harmonic embedding is orientation preserving.

It was shown in [8, 9] that for each holomorphic quadratic differential $\Phi=\phi(z) d z^{2}$ which is not identically zero, there is a complete harmonic open embedding, unique up to conformal transformations, $u: \mathbb{C} \longrightarrow \mathbb{H}^{2}$ such that the Hopf differential of $u$ is exactly $\Phi$.

\subsection{Trajectory structures and measured foliations of the Hopf differentials. Let $\Phi$} be a holomorphic quadratic differential on $\mathbb{C}$, which is given in local coordinate $z$ as $\Phi=$ $\phi(z) d z^{2}$, where $\phi$ is in general a holomorphic function. For any $z_{0} \in \mathbb{C}$ with $\phi\left(z_{0}\right) \neq 0$, there is a choice of a continuous branch of $\sqrt{\phi(z)}$ in a neighborhood $W$ of $z_{0}$. Then for a given base point $z_{*} \in W$ sufficiently close to $z_{0}$, the mapping

$$
\zeta(z)=\int_{z_{*}}^{z} \sqrt{\phi(w)} d w
$$

is univalent in possibly a smaller neighborhood of $z_{0}$ in $W$. This defines local charts on $\{\phi \neq 0\}$ and determines two measured foliations on $\mathbb{C}$ with singularities at the zeros of $\phi$. In particular, the leaves of them are curves given locally by the sets,

$$
\begin{array}{ll}
\Gamma_{\nu}=\{z \in W ; \operatorname{Im}(\zeta(z))=\nu\}, & \nu \in \mathbb{R}, \\
\gamma_{\mu}=\{z \in W ; \operatorname{Re}(\zeta(z))=\mu\}, & \mu \in \mathbb{R} .
\end{array}
$$

Each $\Gamma_{\nu}$ and $\gamma_{\mu}$ is called a horizontal trajectory and vertical trajectory, respectively. The foliations formed by these curves are called horizontal foliation and vertical foliation correspondingly. Obviously, the two foliations have orthogonal leaves. Furthermore, if $z_{0} \in \mathbb{C}$ is a zero of order $m$ of $\phi$, then there are $m+2$ horizontal trajectories, as well as vertical ones, limiting to $z_{0}$. Therefore, the horizontal and vertical foliations are in fact measured foliations with singularities at the zeros of $\Phi$ with natural measures given by $|d \operatorname{Im} \zeta|$ and $|d \operatorname{Re} \zeta|$, respectively. We refer the reader to [1] for the definition of measured foliation on Riemann surface in the general situation.

1.3. The canonical trees associated to the Hopf differentials. For each $\Phi$, the leaf space of the measured foliation given by the vertical trajectories has a special 1-dimensional 
structure called $\mathbb{R}$-tree [10, 11, 12. In this article, we shall call it the $\mathbb{R}$-tree associated to $\Phi$ and denote it by $T_{\Phi}$, or simply by $T$.

A trajectory that tends to a zero of $\Phi$ at least in one direction is called a critical trajectory. Each connected domain of the complement of all critical vertical trajectories is sometimes called a vertical domain, which is foliated by non-critical vertical trajectories.

In the particular case of a quadratic differential $\Phi=P(z) d z^{2}$ for a polynomial $P$ of degree $n$ on $\mathbb{C}$, according to the global structural theorem of meromorphic quadratic differentials on compact Riemann surfaces 4], there are generically $2 n+1$ vertical domains. Among these domains, $n+2$ are called end domains and at most $n-1$ are strip domains. The definition of these two types of domains is given as follows.

For each vertical domain $\Omega$, a canonical mapping $z \mapsto \zeta(z)$ sends $\Omega$ one-to-one onto one of the following domains in $\mathbb{C}$,

(1) a half plane, in such case $\Omega$ is called an end domain;

(2) a vertical strip, $\{\zeta \in \mathbb{C} ; a<\operatorname{Re}(\zeta)<b\}, a, b \in \mathbb{R}$, in such case $\Omega$ is called a strip domain.

Note that the distance on the $\mathbb{R}$-tree $T$ can be realized in the following way. Let $p, q$ be two points on $T$ represented by two leaves $\gamma_{1}$ and $\gamma_{2}$, respectively. One may construct a sequence of arcs from $\gamma_{1}$ to $\gamma_{2}$ such that each arc lies either in a horizontal trajectory or a vertical one. The distance $d_{T}(p, q)$ is given by the sum of the lengths of the horizontal arcs. In particular, if the straight line between $p$ and $q$ on the $\mathbb{R}$-tree can be represented by a single horizontal trajectory in an end domain, then $d_{T}(p, q)$ equals the $\Phi$-length of that horizontal trajectory.

Consequently, one can see that the associated $\mathbb{R}$-tree $T$ has $n+2$ infinite edges corresponding to the $n+2$ end domains, at most $n-1$ finite edges corresponding to the strip domains, and with $n$ vertices corresponding to the zeros counted with multiplicity.

For the special case that $\Phi=\left(z^{2 m}-c z^{m-1}\right) d z^{2}$ with generic $c \in \mathbb{C}$, we see that there are $m+1$ non-degenerate vertices corresponding to the roots of $z^{m+1}-c$ and $m-1$ vertices degenerated to a single vertex corresponding to $z=0$ if $m \geq 3$. The tree $T$ will further degenerate if $c$ is real; and for $c=0$, it will completely degenerate to a single vertex. More precisely, we have 
Lemma 1.1. Let $T$ be the $\mathbb{R}$-tree associated to the quadratic differential $\Phi=\left[z^{2 m}-(a+\right.$ $\left.i b) z^{m-1}\right] d z^{2}, a+i b \in \mathbb{C}$, and $m \geq 1$.

(1) If $m \geq 2$ and $b \neq 0$, then $T$ has $m+1$ non-degenerate vertices each incident with two infinite edges; and all of these vertices are adjacent to a unique vertex, which is nondegenerate for $m=2$ and degenerate otherwise, by finite edges of equal length given by $\pi|b| /(2(m+1))$.

(2) If $m=1$ and $b \neq 0$, then $T$ has 2 non-degenerate vertices each incident with two infinite edges; and they are connected by a finite edge of length $\pi|b| / 2$.

(3) If $m \geq 1$ and $b=0$, then $T$ has a unique vertex incident with $2 m+2$ infinite edges.

Remark 1.2. In the case (2), if we take the mid-point of the 2 vertices as the center of the $\mathbb{R}$-tree, then the vertices are in distance $\pi|b| / 4$ to this center. This is exactly the same value given by the formula in the case (1) with $m=1$. An illustration is given in Figure 1.
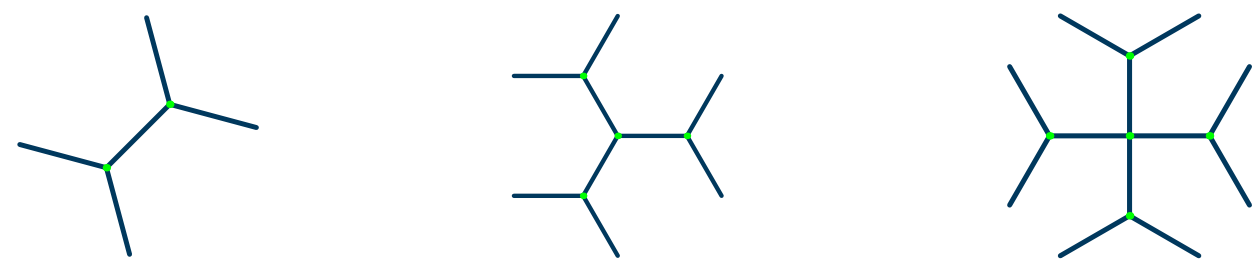

Figure 1: $m=1,2,3$ from left to right.

Remark 1.3. If we consider the associated real tree to the leaf space of the measured foliation given by horizontal trajectories instead of vertical ones, we have another $\mathbb{R}$-tree associated to $\Phi$. One sees that the same is true for this tree except that the common length of the finite edges becomes $\pi|a| /(2(m+1))$. This tree will degenerate when $c$ is pure imaginary.

Proof. By the argument before the Lemma, $T$ has $2 m+2$ infinite edges corresponding to the $2 m+2$ end domains and has $m+1$ non-degenerate vertices. If $m \geq 2$, then $T$ also has a vertex with multiplicity $m-1$. So we only need to show that each non-degenerate vertex is incident with two infinite edges and calculate the distance between the non-degenerate vertices and the vertex with multiplicity.

For any $m \geq 1$, let $\eta$ be a $(m+1)$-root of $c=a+i b$ and $\omega$ be a primitive $(m+1)$-root of unity. Then the roots of $z^{m+1}-c$ are exactly $\left\{\eta, \omega \eta, \ldots, \omega^{m} \eta\right\}$. For any fixed $k=0,1, \ldots, m$, 
there is a wedge with vertex at $z=0$, containing the path $z_{k}(t)=t \omega^{k} \eta, t \in(0,1)$, but no other zero of $\Phi$. Therefore, one can find a domain $\Omega_{k}$ containing the path $z_{k}(t)$ which is contained in a strip domain of $\Phi$. Choosing a branch of the natural parameter

$$
\zeta_{k}(z)=\int^{z} \sqrt{z^{m-1}\left(z^{m+1}-c\right)} d z
$$

on $\Omega_{k}$ and taking limits at $t \rightarrow 0$ and $t \rightarrow 1$, one sees that

$$
\zeta_{k}\left(\omega^{k} \eta\right)-\zeta_{k}(0)= \pm i c \int_{0}^{1} \sqrt{t^{m-1}\left(1-t^{m+1}\right)} d t .
$$

This implies that the horizontal $\Phi$-distance between the root $\omega^{k} \eta$ and 0 is given by $|b| \int_{0}^{1} \sqrt{t^{m-1}\left(1-t^{m+1}\right)} d t=$ $\pi|b| /(2(m+1))$. This proves that, in the $\mathbb{R}$-tree, the vertices corresponding to the roots of $z^{m+1}-c$ are adjacent to the vertex corresponding to $z=0$ by a finite arc of length $\pi|b| /(2(m+1))$ if $m \geq 2$. If $m=1$, then the same calculation shows that the 2 vertices are connected by a finite arc of length $2 \times \pi|b| /(2(m+1))=\pi|b| / 2$.

Therefore, in the case that $m \geq 2$, the vertex corresponding to $z=0$ with multiplicity $m-1$ already has $m+1$ finite edges incident with the $m+1$ non-degenerate vertices, and hence it is not incident with any other edges of $T$. Since there are $2 m+2$ infinite edges, each of the vertices corresponding to the roots of $z^{m+1}-c$ must be incident with 2 infinite edges. The case that $m=1$ is trivial, since $z=0$ is not even a critical point of $\Phi$. Finally, by counting the multiplicity of the vertices and the number of edges, we see that there is no other edge of $T$ and the proof is completed.

\section{Distance estimates}

In [3], it was shown that the image of an horizontal trajectory far from zeroes is exponentially close to the geodesic connecting the end points in the $\Phi$-distance of the trajectory. However, this is not enough in our discussion about the image of the harmonic map. In fact, we need to show that the difference between the lengths of the image and the geodesic is actually tending to zero as the $\Phi$-distance is going to infinity. Note that we need more than just the ratio tending to 1 as in Proposition 2.2 of [7]. 
Lemma 2.1. Let $\gamma_{R}, R>0$ be a family of curves in the hyperbolic 2-space such that, as $R \rightarrow+\infty, L\left(\gamma_{R}\right)=O(R)$ and $\left\|k_{g}\right\|\left(\gamma_{R}\right)=O\left(e^{-a R}\right)$ for some $a>0$, where $L\left(\gamma_{R}\right)$ is the length of $\gamma_{R}$ and $\left\|k_{g}\right\|\left(\gamma_{R}\right)$ is the supremum of the absolute value of the geodesic curvature of $\gamma_{R}$. Then the distance $d(R)$ between the end points of $\gamma_{R}$ satisfies $d(R)=L\left(\gamma_{R}\right)+o(R)$ as $R \rightarrow+\infty$.

Proof. For a sufficiently large fixed $R>0$, we work in the Fermi coordinates $(u, v)$ with respect to the geodesic $\gamma_{R}^{*}$ passing through the end points $\gamma_{R}(0)$ and $\gamma_{R}(l)$, where $l=L\left(\gamma_{R}\right)$ is the length of $\gamma_{R}$. That is, $\gamma_{R}:[0, l] \rightarrow \mathbb{H}^{2}$ is parametrized by arc-length, and $\gamma_{R}^{*}$ is given by $v \equiv 0$.

By Lemma 3.1 in [3], there exists a constant $C>0$ such that, for sufficiently small $\epsilon>0$, $\left\|k_{g}\right\|\left(\gamma_{R}\right)<\epsilon$ implies $d\left(\gamma_{R}, \gamma_{R}^{*}\right)<C \epsilon$. That is,

$$
\left|v\left(\gamma_{R}(s)\right)\right|<C \epsilon \quad \text { for all } s \in[0, l]
$$

As in [3], we have

$$
u^{\prime 2} \cosh ^{2} v+v^{\prime 2} \equiv 1
$$

and

$$
k_{g}^{2}=\cosh ^{2} v\left(u^{\prime \prime}+2 u^{\prime} v^{\prime} \tanh v\right)^{2}+\left(v^{\prime \prime}-u^{\prime 2} \cosh v \sinh v\right)^{2} .
$$

Let

$$
h_{1}=u^{\prime \prime} \cosh v+2 u^{\prime} v^{\prime} \sinh v, \quad h_{2}=v^{\prime \prime}-u^{\prime 2} \cosh v \sinh v .
$$

Then $h_{1}^{2}+h_{2}^{2}=k_{g}^{2}$. On the other hand, differentiation of (2.2) gives $h_{1} u^{\prime} \cosh v+h_{2} v^{\prime}=0$, i.e., $\left(h_{1}, h_{2}\right)$ is orthogonal to $\left(u^{\prime} \cosh v, v^{\prime}\right)$. Therefore, we must have $\left(h_{1}, h_{2}\right)= \pm\left|k_{g}\right|\left(v^{\prime},-u^{\prime} \cosh v\right)$. Consequently, we have

$$
\left|h_{1}\right| \leq \epsilon\left|v^{\prime}\right| \quad \text { and } \quad\left|h_{2}\right| \leq \epsilon\left|u^{\prime}\right| \cosh v
$$

We may assume that $u(0)=0$. Then $d(R)=u(l)$. Applying Poincaré inequality to $u^{\prime}(s)-$ $u(l) / l$ and $v^{\prime}(s)$, we conclude that there is a constant $C_{1}>0$ such that

$$
\int_{0}^{l} u^{\prime 2} \leq \frac{d(R)^{2}}{l}+C_{1} l^{2} \int_{0}^{l}\left(u^{\prime \prime}\right)^{2}
$$

and

$$
\int_{0}^{l} v^{\prime 2} \leq C_{1} l^{2} \int_{0}^{l}\left(v^{\prime \prime}\right)^{2} .
$$


Therefore, (2.1), (2.2), (2.4) and (2.5) imply

$$
\int_{0}^{l} u^{\prime 2} \leq \frac{d(R)^{2}}{l}+C_{2} l^{2} \epsilon^{2} \int_{0}^{l} v^{\prime 2}
$$

for some constant $C_{2}>0$. Similarly, we have from (2.1), (2.2), (2.4) and (2.6) that

$$
\int_{0}^{l} v^{\prime 2} \leq C_{3} l^{2} \epsilon^{2} \int_{0}^{l} u^{\prime 2}
$$

for some constant $C_{3}>0$. Putting this into (2.7), we have

$$
\int_{0}^{l} u^{\prime 2} \leq \frac{d(R)^{2}}{l}+C_{2} C_{3} l^{4} \epsilon^{4} \int_{0}^{l} u^{\prime 2} .
$$

By the assumption on the geodesic curvature $\left\|k_{g}\right\|$, we may choose $\epsilon=O\left(e^{-a R}\right)$. Then, together with $l=O(R)$, one has $C_{2} C_{3} l^{4} \epsilon^{4} \leq \epsilon^{2}<1$ for sufficiently large $R$. Hence, (2.9) gives

$$
\int_{0}^{l} u^{\prime 2} \leq \frac{1}{\left(1-\epsilon^{2}\right)} \frac{d(R)^{2}}{l}=\left[1+O\left(e^{-2 a R}\right)\right] \frac{d(R)^{2}}{l} .
$$

On the other hand, from (2.1) and (2.2), we have

$$
l=\int_{0}^{l} u^{\prime 2} \cosh ^{2} v+v^{\prime 2} \leq\left(1+C^{2} \epsilon^{2}\right) \int_{0}^{l} u^{\prime 2}+\int_{0}^{l} v^{\prime 2} .
$$

Together with the estimates (2.8) and (2.10), this gives

$$
l \leq\left[1+O\left(e^{-a R}\right)\right] \frac{d(R)^{2}}{l} .
$$

Therefore, $l=O(R)$ implies

$$
d(R) \geq l\left(1+O\left(e^{-a R}\right)\right)^{-1}=l-l \cdot O\left(e^{-a R}\right) \geq l-O\left(e^{-a R / 2}\right) .
$$

As it is trivial that $l \geq d(R)$, we have shown that $d(R)=L\left(\gamma_{R}\right)+o(R)$.

From Lemma 2.1, we have the following corollary on the asymptotic behavior of harmonic maps.

Corollary 2.2. Let $\Gamma_{R}, R>0$, be a family of horizontal trajectories of a holomorphic quadratic differential $\Phi$ with $\Phi$-length equal to $L$. If the $\Phi$-distance of $\Gamma_{R}$ to every zero of $\Phi$ tends to infinity as $R \rightarrow \infty$, then the images $u\left(\Gamma_{R}\right)$ of $\Gamma_{R}$ under the unique harmonic embedding $u: \mathbb{C} \rightarrow \mathbb{H}^{2}$ corresponding to $\Phi$ approaches a boundary geodesic arc of length $2 L$ of the image set $u(\mathbb{C})$ as $R \rightarrow+\infty$. 
Proof. Let us write $\gamma_{R}=u\left(\Gamma_{R}\right)$ and $d_{\phi}(R)$ for the minimal $\Phi$-distance of $\Gamma_{R}$ to zeroes of $\Phi$. First of all, the arguments of Lemma 3.2 and 3.4 of [3] imply that $\gamma_{R}$ approaches the boundary geodesic of $u(\mathbb{C})$. So we only need to calculate its length. Let $e^{2 w}$ be the $\partial$-energy density of $u$ with respect to the $\Phi$-metric in its natural coordinates, i.e., $\Phi=d z^{2}=(d x+i d y)^{2}$. Then, by Formula (3.6) of [3], we obtain

$$
L\left(\gamma_{R}\right)=\int_{\Gamma_{R}} \sqrt{e^{2 w}+e^{-2 w}+2} d x=\int_{\Gamma_{R}}\left(2+4 \sinh \frac{w}{2}\right) d x .
$$

The exponential decay estimate of [2] then implies that

$$
L\left(\gamma_{R}\right)=2 L+O\left(e^{-a_{1} d_{\Phi}(R)}\right)
$$

for some constant $a_{1}>0$. On the other hand, the estimate as in the proof of Lemma 3.2 in [3] shows that

$$
\left\|k_{g}\right\|\left(\gamma_{R}\right)=O\left(e^{-a_{2} d_{\Phi}(R)}\right)
$$

for some constant $a_{2}>0$. Therefore, by Lemma 2.1, we conclude that the distance between the end points of $\gamma_{R}$ is equal to $2 L+o(R)$ as $R \rightarrow \infty$. Therefore, by letting $R \rightarrow \infty$, we have the desired result.

\section{IMAGE OF HARMONIC MAPS}

In this section, we prove our main result on the explicit determination of the image of the harmonic embedding with suitable symmetry. We are interested in a harmonic embedding $u$ from $\mathbb{C}$ into $\mathbb{H}^{2}$ such that $u$ is equivariant under the group $\mathbb{Z}_{k}$ by rotations and its image is an ideal polygon with $2 k$ vertices for any integer $k \geq 2$. In some sense, $u$ has half of the symmetry of a regular polygon. Note that our symmetry requirement is not just on the image set but on the map $u$.

According to this requirement, the Hopf differentials of these harmonic embeddings are equivariant under the action $z \mapsto \omega z$ for any $k^{\text {th }}$-root of unity $\omega$ and their coefficients are polynomials of degree $2 k-2$. This immediately implies that the Hopf differentials are of the form $\left[z^{2 m}-(a+i b) z^{m-1}\right] d z^{2}$, where $a+i b \in \mathbb{C}$. For these type of harmonic embeddings, we have the following 
Theorem 3.1. Let $u: \mathbb{C} \rightarrow \mathbb{H}^{2}$ be the unique (up to equivalence) harmonic embedding associated to a quadratic differential equivalent to $\left[z^{2 m}-(a+i b) z^{m-1}\right] d z^{2}$. Then, up to isometry, the image $u(\mathbb{C})$ is the interior of the ideal polygon with vertices given by

$$
\left\{1, e^{i \alpha}, \omega, \omega e^{i \alpha}, \ldots, \omega^{m}, \omega^{m} e^{i \alpha}\right\}
$$

in the unit disc model of $\mathbb{H}^{2}$, where $\omega=e^{2 \pi i /(m+1)}$,

$$
\alpha=\alpha_{m}(\nu)=2 \tan ^{-1}\left(\frac{\sin (\pi /(m+1))}{\cos (\pi /(m+1))+e^{2 \nu}}\right),
$$

and $\nu=\pi|b| /(2(m+1))$ is the common length of the finite edges of the $\mathbb{R}$-tree associated to the quadratic differential given by Lemma 1.1.

Proof. As a harmonic map from a surface is invariant under conformal change of metrics on the surface, we may assume that the Hopf differential of $u$ is in fact given by $\Phi=\left[z^{2 m}-\right.$ $\left.(a+i b) z^{m-1}\right] d z^{2}$. Then, by the symmetry of the quadratic differential $\Phi$ and the uniqueness property of the corresponding complete orientation preserving harmonic embedding, after a composition with an isometry on $\mathbb{H}^{2}$, the harmonic embedding satisfies $u(0)=0$ and the image $u(\mathbb{C})$ is an ideal polygon with vertices given by

$$
\left\{1, e^{i \alpha}, \omega, \omega e^{i \alpha}, \ldots, \omega^{m}, \omega^{m} e^{i \alpha}\right\}
$$

for some $\alpha \in(0,2 \pi /(m+1))$. What we need to do is to determine $\alpha$. We also note that, by the rotation of an angle $-\alpha$, this polygon is equivalent to $\left\{1, e^{i \beta}, \omega, \omega e^{i \beta}, \ldots, \omega^{m}, \omega^{m} e^{i \beta}\right\}$ with $\beta=2 \pi /(m+1)-\alpha$. Therefore, we may assume that $\alpha \in(0, \pi /(m+1))$.

Let $0 \in\left(T, d_{T}\right)$ be the vertex on the associated $\mathbb{R}$-tree not incident with any infinite edge for $m \geq 2$ or the mid-point of the unique pair of vertices for $m=1$ as described in Lemma 1.1. For sufficiently large $L>0$, the set $\left\{q \in T ; d_{T}(q, 0)=L\right\}$ has exactly $2 m+2$ points $\left\{q_{0}, \ldots, q_{2 m+1}\right\} \subset T$ such that each infinite edge contains exactly one $q_{i}$. As the tree $T$ is coming from the trajectories structure of $\Phi$ on the plane, there is a natural induced cyclic order of the set of infinite edges. Assume that $q_{i}$ are labelled in the same cyclic order, for $i \in \mathbb{Z}_{2 m+2}$. Then for each pair of consecutive points $q_{i}, q_{i+1}$, we can find $z_{i}$ and $z_{i+1}^{\prime}$ in $\mathbb{C}$ both contained in a common horizontal trajectory $\Gamma_{i}$ of distance $R$ to zeroes in an end domain, denoted by $E_{i}$, of $\Phi$ such that each $z_{i}$ and $z_{i+1}^{\prime}$ belongs to the vertical trajectories representing 
$q_{i}$ and $q_{i+1}$, respectively. Note that from our choice, $z_{i}$ and $z_{i}^{\prime}$ belong to the same vertical trajectory $\gamma_{i}$ representing $q_{i}$. An illustration is given in Figure 2.

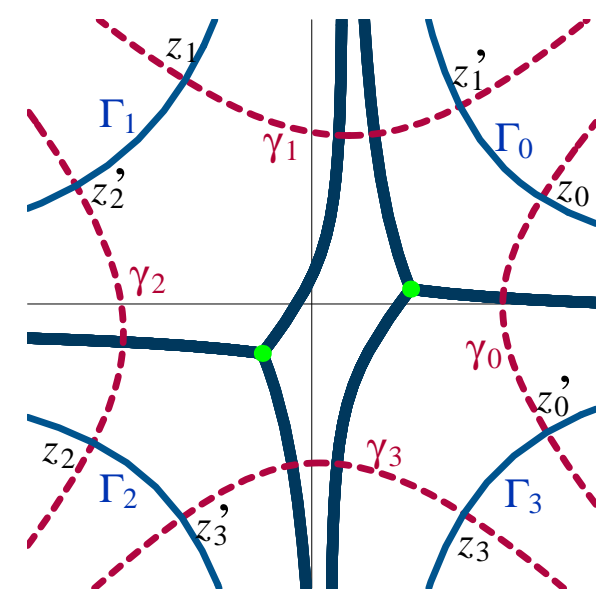

Figure 2.

Up to isometry, we may assume that the image curve of the vertical trajectory representing $q_{0}$ approaches the ideal boundary to the point 1 in the unit disc model of $\mathbb{H}^{2}$ as $L \rightarrow+\infty$. Correspondingly, the image points $u\left(z_{0}\right)$ and $u\left(z_{0}^{\prime}\right)$ of $z_{0}$ both tend to 1 . Then, by the symmetry of $u$ and our assumption, for each $k=0, \ldots, m$, the image curve of the vertical trajectory representing $q_{2 k}$ approaches the ideal boundary point $\omega^{k}=e^{2 \pi k i /(m+1)}$, and the image curve of the vertical trajectory representing $q_{2 k+1}$ approaches the ideal boundary point $\omega^{k} e^{i \alpha}$, respectively, in the unit disc model of $\mathbb{H}^{2}$. In accordance with this, the image points $u\left(z_{2 k}\right)$ and $u\left(z_{2 k}^{\prime}\right)$ tend to $\omega^{k}$, while $u\left(z_{2 k+1}\right)$ and $u\left(z_{2 k+1}^{\prime}\right)$ tend to $\omega^{k} e^{i \alpha}$.

To determine the $\Phi$-length of each $\Gamma_{i}$, we observe from Lemma 1.1, which concerns the tree structure of $\mathbb{R}$-tree $T$ associated to $\Phi$, that $d_{T}\left(q_{i}, q_{i+1}\right)=2 L$ or $2(L-\nu)$, with the value taken alternatingly in $i$, where $\nu=\pi|b| /(2(m+1))$ is the common length of those finite edges of $T$. We first assume that

$$
d_{T}\left(q_{2 k}, q_{2 k+1}\right)=2(L-\nu) \quad \text { and } \quad d_{T}\left(q_{2 k+1}, q_{2 k+2}\right)=2 L
$$

An illustration is given in Figure 3. 


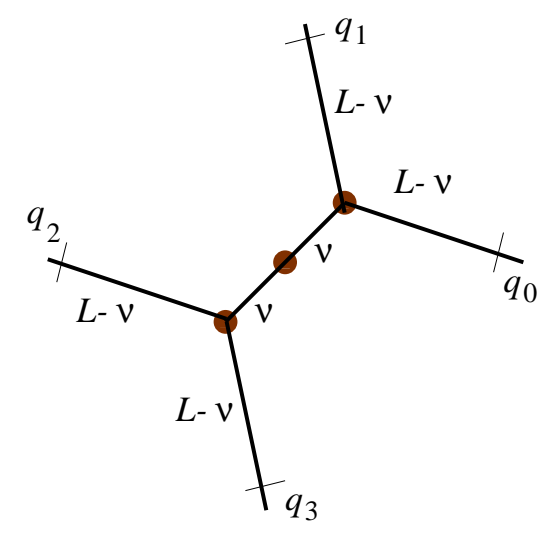

Figure 3.

As $\Gamma_{i}$ is a horizontal trajectory with end points representing $q_{i}$ and $q_{i+1}$, the $\Phi$-length of $\Gamma_{i}$ is exactly equal to $d_{T}\left(q_{i}, q_{i+1}\right)$. Therefore, for $k=0, \ldots, m$,

$$
L_{\Phi}\left(\Gamma_{2 k}\right)=2(L-\nu) \quad \text { and } \quad L_{\Phi}\left(\Gamma_{2 k+1}\right)=2 L
$$

On the other hand, the vertical trajectory $\gamma_{i}$ representing $q_{i}$ is mapped to a curve of finite length in $\mathbb{H}^{2}$. Indeed, using natural coordinates of $\Phi$ in an end domain containing $\gamma_{i}$ with respect to the vertical trajectories system, the length of the image curve is given by

$$
l_{i}=L_{\mathbb{H}^{2}}\left(u\left(\gamma_{i}\right)\right)=\int_{-\infty}^{+\infty} \sqrt{e^{2 w}+e^{-2 w}-2} d y=\int_{-\infty}^{+\infty} 2 \sinh w d y,
$$

where $w$ as in the proof of Corollary 2.2. As $\gamma_{i}$ is at least a $\Phi$-distance of $L-\nu$ away from zeroes, the exponential decay estimate of $w$ implies that for some $y_{0}$ and $a>0$,

$$
\begin{aligned}
l_{i} & \leq C\left[\int_{-y_{0}}^{y_{0}} e^{-a L} d y+\left(\int_{-\infty}^{-y_{0}}+\int_{y_{0}}^{+\infty}\right) e^{-a(L+|y|) / \sqrt{2}} d y\right] \\
& \leq O\left(e^{-a L / \sqrt{2}}\right) .
\end{aligned}
$$

Therefore, $l_{i}$ are finite and tends to zero as $L \rightarrow+\infty$.

Let $\zeta_{2 k}$ be a point on the intersection of $u\left(\gamma_{2 k}\right)$ and the ray from 0 to $\omega^{k}$ in the Poincaré disc. Similarly, let $\zeta_{2 k+1}$ be a point on the intersection of $u\left(\gamma_{2 k+1}\right)$ and the ray from 0 to $\omega^{k} e^{i \alpha}$. Now consider the polygon in $\mathbb{H}^{2}$ with vertices $0, \zeta_{0}, \zeta_{1}$, and $\zeta_{2}$. Note that, since $l_{i} \rightarrow 0$ as $L \rightarrow+\infty$, the distance between $\zeta_{i}$ and $u\left(z_{i}\right)$ or $u\left(z_{i}^{\prime}\right)$ also tends to zero. An illustration is given in Figure 4. 


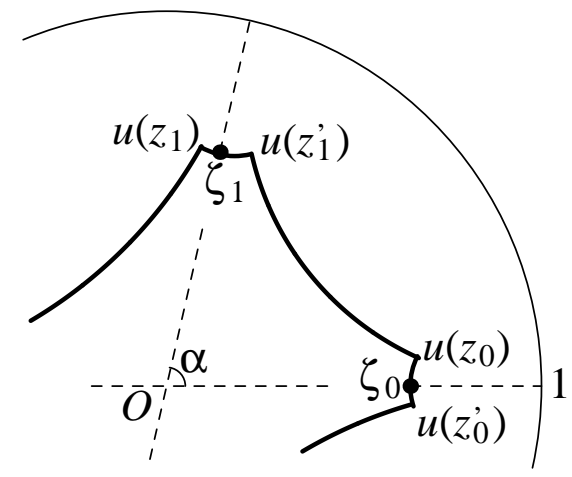

Figure 4 .

On the other hand, by letting $R \rightarrow+\infty$, Corollary 2.2 implies that the image $u\left(\Gamma_{i}\right)$ of the horizontal arc $\Gamma_{i}$ connecting $z_{i}$ and $z_{i}^{\prime}$ approaches a boundary geodesic arc of length $2(L-\nu)$ and $2 L$, alternatingly in $i$. All together, we conclude that, as $L \rightarrow+\infty$,

$$
d_{\mathbb{H}^{2}}\left(\zeta_{0}, \zeta_{1}\right)=4(L-\nu)+o(L) \quad \text { and } \quad d_{\mathbb{H}^{2}}\left(\zeta_{1}, \zeta_{2}\right)=4 L+o(L)
$$

Let $x_{1}=x_{1}(L)=d_{\mathbb{H}^{2}}\left(\zeta_{0}, 0\right)$ and $x_{2}=x_{2}(L)=d_{\mathbb{H}^{2}}\left(\zeta_{1}, 0\right)$. Then, by symmetry, $d_{\mathbb{H}^{2}}\left(\zeta_{2}, 0\right)$ is also equal to $x_{1}$. Hence from the cosine rule of the hyperbolic plane, we have

$$
\cosh (4(L-\nu)+o(L))=\cosh x_{1} \cosh x_{2}-\sinh x_{1} \sinh x_{2} \cos \alpha
$$

and

$$
\cosh (4 L+o(L))=\cosh x_{1} \cosh x_{2}-\sinh x_{1} \sinh x_{2} \cos (2 \pi /(m+1)-\alpha) .
$$

It is easy to see from these identities that $\lim _{L \rightarrow+\infty}\left(e^{-4 L} \sinh x_{1} \sinh x_{2}\right)$ exists and is non-zero. Let us denote

$$
A=\left[4 \lim _{L \rightarrow+\infty}\left(e^{-4 L} \sinh x_{1} \sinh x_{2}\right)\right]^{-1} .
$$

Then multiplying by $\left(\sinh x_{1} \sinh x_{2}\right)^{-1}$ to the above equations and letting $L \rightarrow+\infty$, one concludes that

$$
\sqrt{A} e^{-2 \nu}=\sin \frac{\alpha}{2} \quad \text { and } \quad \sqrt{A}=\sin \left(\frac{\pi}{m+1}-\frac{\alpha}{2}\right) .
$$

It is easy to solve the above and obtain

$$
\tan \frac{\alpha}{2}=\frac{\sin (\pi /(m+1))}{\cos (\pi /(m+1))+e^{2 \nu}},
$$

which is the desired result. 
In the case that

$$
d_{T}\left(q_{2 k}, q_{2 k+1}\right)=2 L \quad \text { and } \quad d_{T}\left(q_{2 k+1}, q_{2 k+2}\right)=2(L-\nu)
$$

the same calculation shows that the angle is given by

$$
\tan \frac{\alpha}{2}=\frac{\sin (\pi /(m+1))}{\cos (\pi /(m+1))+e^{-2 \nu}} .
$$

The angle obtained in this formula belongs to $[\pi /(m+1), 2 \pi /(m+1))$, which is equivalent to the one in previous formula by the transformation $\alpha \mapsto 2 \pi /(m+1)-\alpha$.

In [7, a harmonic map was constructed with image equal to a regular ideal polygon of 4 vertices and Hopf differential is given by $\left(z^{2}+i b\right) d z^{2}$ for some real number $b \in \mathbb{R}$. From our theorem, one in fact has

Corollary 3.2. The harmonic diffeomorphism constructed in Proposition 1.6 of [7] is a unique, up to equivalence, complete orientation preserving harmonic embedding with Hopf differential $z^{2} d z^{2}$

Proof. When $m=1$, the theorem implies that the image of the harmonic map is equivalent to $\left\{1, e^{i \alpha},-1,-e^{i \alpha}\right\}$ with $\alpha=2 \tan ^{-1}\left(e^{-2 \nu}\right)$. So $\alpha=\pi / 2$ if and only if $\nu=0$. Since $\nu=$ $2 \pi|b| /(m+1)$, we conclude that $b=0$.

Finally, let us finish the paper by a couple of remarks.

Remark 3.3. The fact that the image ideal polygon depends only on $|b|$ but not $b$ can be easily seen from the fact that $\left[z^{2 m}-(a+i b) z^{m-1}\right] d z^{2}$ is equivalent to $\left[z^{2 m}+(a+i b) z^{m-1}\right] d z^{2}$.

Remark 3.4. Let $P_{\alpha}$ be the equivalence class of the ideal polygon

$$
\left\{1, e^{i \alpha}, \omega, \omega e^{i \alpha}, \ldots, \omega^{m}, \omega^{m} e^{i \alpha}\right\}
$$

Then the mapping $\alpha \mapsto P_{\alpha}$ from $(0,2 \pi /(m+1))$ to all such equivalence classes is two-to-one except at $\alpha=\pi /(m+1)$, which maps to the regular ideal polygon. Therefore from the proof, one may define, for each $\Phi=\left[z^{2 m}-(a+i b) z^{m-1}\right] d z^{2}$, the angle function by

$$
\alpha(b)=2 \tan ^{-1}\left(\frac{\sin (\pi /(m+1))}{\cos (\pi /(m+1))+e^{b \pi /(m+1)}}\right),
$$


that is, by the same formula without taking absolute value of $b$ as in $\nu=\pi|b| /(2(m+1))$. This angle function $\alpha$ is a bijection from $\mathbb{R}$ to $(0,2 \pi /(m+1))$. Thus, the mapping $b \mapsto P_{\alpha(b)}$ behaves similarly. This gives a 2-fold covering except $b=0$ for each fixed $a$ and is consistent with the previous remark.

Note that if we let the angle $\alpha$ run through the whole interval $(0,2 \pi /(m+1))$, the ideal polygon $\left\{1, e^{i \alpha}, \omega, \omega e^{i \alpha}, \ldots, \omega^{m}, \omega^{m} e^{i \alpha}\right\}$ runs over the set of all possible equivalence classes of polygons except the regular polygon twice and once at the regular idea polygon. Then for each fixed $a, \alpha: \mathbb{R} \rightarrow(0,2 \pi /(m+1))$ is a bijection and the corresponding ideal polygon with vertices $\left\{1, e^{i \alpha}, \omega, \omega e^{i \alpha}, \ldots, \omega^{m}, \omega^{m} e^{i \alpha}\right\}$ runs through the set of all possible equivalent classes of ideal polygons except the regular idea polygon twice and once at the regular idea polygon as $b$ run through $\mathbb{R}$ once.

\section{REFERENCES}

[1] T. Au, L. F. Tam and T. Y.-H. Wan, Hopf differentials and the images of harmonic maps, Comm. Anal. Geom. 10 (2002), 515-573.

[2] Z. C. Han, Remarks on the geometric behavior of harmonic maps between surfaces, 57-66, Elliptic and parabolic methods in geometry, papers from the workshop held at the University of Minnesota, Minneapolis, Minnesota, May 23-27, 1994, Ed. B. Chow, R. Gulliver, S. Levy, \& J. Sullivan, A K Peters, Wellesley, MA, 1996.

[3] Z. Han, L. F. Tam, A. Treibergs and T. Wan, Harmonic maps from the complex plane into surfaces with nonpositive curvature, Comm. Anal. Geom. 3 (1995), 85-114.

[4] J. A. Jenkins, Univalent functions and conformal mappings, 2nd ed., Springer, Berlin, 1965.

[5] Y. N. Minsky, Harmonic maps, length, and energy in Teichmüller space, J. Differential Geom. 35 (1992), $151-217$.

[6] R. Schoen, The role of harmonic mappings in rigidity and deformation problems, Complex Geometry (Osaka, 1990), 179-200, Lecture Notes in Pure and Appl. Math. 143, Dekker, New York, 1993.

[7] Y. Shi and L. F. Tam, Harmonic maps from $\mathbb{R}^{n}$ to $\mathbb{H}^{m}$ with symmetry, Pacific J. Math. 202 (2002), 227-256.

[8] L. F. Tam and T. Y.-H. Wan, Harmonic diffeomorphisms into Cartan-Hadamard surfaces with prescribed Hopf differentials, Comm. Anal. Geom. 2 (1994), 593-625.

[9] T. Y.-H. Wan and T. Au, Parabolic constant mean curvature spacelike surfaces, Proc. Amer. Math. Soc. 120 (1994), 559-564.

[10] M. Wolf, Harmonic maps from surfaces to $\mathbb{R}$-trees, Math. Z. 218 (1995), 577-593.

[11] M. Wolf, On realizing measured foliations via quadratic differentials of harmonic maps to $\mathbb{R}$-trees, J. Anal. Math. 68 (1996), 107-120.

[12] M. Wolf, Measured foliations and harmonic maps of surfaces, J. Differential Geom. 49 (1998), 437-467.

Department of Mathematics

The Chinese University of Hong Kong

Shatin, Hong Kong

CHINA

E-mail addresses: tomwan@math.cuhk.edu.hk, thomasau@cuhk.edu.hk 